\title{
COVID-19 and Dengue infection in Bangladesh: a case of coinfection where hemoptysis as first presentation
}

\author{
Mohammad Ashraful Amin ${ }^{1}$, Md. Taufiqul Islam ${ }^{1}$, Ishtiakul Islam Khan ${ }^{1}$, Zahid Hasan \\ Khan $^{1}$, Firdausi Qadri ${ }^{1}$, and Ashraful Islam Khan ${ }^{2}$ \\ ${ }^{1} \mathrm{ICDDRB}$ \\ ${ }^{2}$ International Centre for Diarrhoeal Disease Research Bangladesh
}

November 25, 2021

\begin{abstract}
Bangladesh recently faced large outbreaks of both COVID-19 and Dengue fever. A 28-year-old woman suffered from symptoms including hemoptysis as first presentation followed by high-grade fever, sore throat, and fatigue. SARS-CoV-2 was confirmed by RT-PCR and also diagnosed dengue later on.COVID-19 and dengue fever could be a harmful combination.
\end{abstract}

\section{Introduction}

COVID-19 infection is caused by the Severe Acute Respiratory Syndrome Coronavirus 2 (SARS-CoV-2) virus, which has already propagated to over 210 nations and territories throughout the worldwide. The World Health Organization (WHO) has declared it a pandemic emergency due to the rise of COVID-19 cases in March 2020. COVID-19 was confirmed for the first time in Bangladesh on March 8, 2020 (1). Till date, globally 190 million cases along with over 4 million deaths have been recorded due to COVID-19. COVID-19 infection can have a wide range of symptoms, from mild to severe acute respiratory syndrome requiring respiratory support (2). Hemoptysis is prevalent in about up to 13 percent of pulmonary embolism patients and seldom seen among COVID-19 patients (3). Aside from the continuing pandemic, dengue is predominant for the last decades and caused high morbidity and mortality. Bangladesh has been hit by dengue fever every year, and COVID-19 pandemic started in Bangladesh from March, 2020 (4). Dengue fever is a viral infection caused by four kinds of Flaviviridae viruses (DENV-1, DENV-2, DENV-3, and DENV-4). Infected Aedes aegypti and Aedes albopictus female mosquitoes are the most common carriers of these viruses(5). However, dengue and COVID-19 might be a hazardous combination. According to clinician observation, many cases have been detected in different hospitals in Bangladesh. In this report, we have presented a case of COVID-19 and dengue co-infection in a person in Bangladesh, where the patient presented hemoptysis as first presentation.

\section{Case report}

The patient was 28-year old women with no history of any significant co-morbidities. She had a history of contact with COVID-19 cases, but no history of travel outside Dhaka or other country for last six months. Her relative was diagnosed as COVID-19 on 24th June, 2021 with whom she lives. On June 26, 2021, the case felt a mild cough followed by blood in cough one time. As her family history was positive, she went for testing for RT-PCR for SARS-CoV-2 and became positive on $28^{\text {th }}$ June, 2021 and was in isolation at home and in addition, the dengue RDT, Ag NS1 (ICT) was negative. Her symptoms included dry cough, sore throat, fever $\left(100^{\circ} \mathrm{F}\right)$ and shivering at night. She had hemoptysis for two times on June 28,2021 , which consisted of scanty amount of blood. On the next day she had fever (highest body temperature $102^{\circ} \mathrm{F}$ ), with dry cough constantly, 
sore throat followed by fatigue. She monitored her vital signs regularly and her oxygen saturation was 9899\%. Routine blood tests such as, complete blood count, serum creatinine, D-dimer, serum ferritin done and test results were normal (Table 1). Her chest x-ray also revealed no abnormalities. The patient started medications from June 29, 2021 which included Paracetamol 500mg 1tab 8hourly, Azithromycin 500mg once at night, Montelukast 10mg once at night, Desloratadine $10 \mathrm{mg}$ once in the morning and Multivitamin once in the morning.

On 30th June 2021, physical examination indicated normal vital signs. On auscultation of the lungs, a vesicular breath sound was heard, along with bilateral basal coarse crepitation. There was sign of red rash without itchiness on both upper limbs of the body. Then again on $6^{\text {th }}$ July, investigations were conducted; laboratory reports showed hemoglobin $11.5 \mathrm{~L} \mathrm{~g} / \mathrm{dL}$, lymphocyte count $23.2 \%$, neutrophil $72.3 \%$, total leucocyte count $5.13 \mathrm{~L} \times 10^{\wedge} 9 / \mathrm{L}$, red cell count $4.40 \times 10^{\wedge} 12 / \mathrm{L}$, mean cell hemoglobin $26.1 \mathrm{pg}$, platelet count $175 \times 10^{\wedge}$ $9 / \mathrm{L}$ and dengue IgM was positive, CRP was 0.34 . On $6^{\text {th }}$ July mild respiratory distress occurred with dry cough and she was given Salmeterol Xinafoate and Fluticasone Propionate (25/125) two times a day and Deflazacort $6 \mathrm{mg}$ started 2-tab 8 hourly.

Thoracic computed tomography $(\mathrm{CT})$ done on $6^{\text {th }}$ July, 2021 revealed subtle ground glass and consolidative opacities were noted in different segments of both lungs, predominantly in a peripheral location with basal distribution, nearly $30 \%$ of lung involvement was present. Also sub centimetric mediastinal lymph nodes was also present (Figure 1). These findings were reported in accordance with alveolar hemorrhage.

On $8^{\text {th }}$ July 2021, the patient was in moderate respiratory distress. Her temperature was 100 degF, blood pressure 120/70 $\mathrm{mmHg}$, pulse rate $96 \mathrm{bpm}$ (regular) and oxygen saturation on pulse-oximetry $92-95 \%$ without oxygen. Basal crepitation was found on lung auscultation. She was admitted to the Green life medical college hospital, Dhaka for respiratory distress. Then she was immediately started on stat dose of Remdesivir 200mg IV, Hydrocortisone 100mg 8 hourly, nebulization with Ipratropium Bromide plus Salbutamol 8 hourly, Montelukast 10mg at night, Paracetamol 500mg as required, Moxifloxacin 400mg once at night. She did not require any oxygen during hospitalization. On $9^{\text {th }}$ July 2021 , the patient was stable with ongoing treatment and her blood and urine culture, both reports revealed no bacterial growth and her oxygen saturation 95\%-98\%. On the 10th July 2021, she was discharged from hospital in a relatively stable condition, with the above-mentioned drugs and advice of respiratory exercise. Hemoptysis was her first symptom on June $26^{\text {th }}$, and she experienced twice the next day, in the morning and at night. Actually, this patient was treated at home initially from June 29 to July 7, 2021 and she had self -medication at home. Also due to the red rash, a dengue test was again performed on July 6, 2021 and the results were positive for dengue IgM. Then on the 8th July 2021, she was admitted to the hospital, and on the 10th July 2021, she was discharged. On July 15, 2021, she recovered and became symptom-free. During follow-upon July 29,2021, a chest CT revealed full clearance of the ground glass opacities, and an RT PCR of a nasopharyngeal swab for SARS-CoV-2 was negative, indicating that her clinical condition had improved There was no further occurrence of hemoptysis after recovery from COVID-19.

\section{Discussion}

COVID-19 and dengue have a spectrum of disease with overlap in clinical manifestations. Here, we reported a confirmed case of COVID-19 along with coinfection by dengue. Hemoptysis was the initial and only indication of COVID-19 infection in this patient with dengue fever, which was atypical. Hemoptysis can range from mild to severe from a smear of blood in the sputum to a life-threatening hemorrhage (6). The majority of dengue infection patients have bleeding, shock, organ failure, and metabolic acidosis as a result of their illness. It is unusual for lower respiratory tract to be involved. Dengue fever is a potentially fatal disease characterized by pulmonary capillaritis, which causes diffuse alveolar hemorrhage (DAH) which can lead to hemoptysis (7). It may be the symptom of a number of diseases such as tuberculosis, malignancy, bronchiectasis, and pneumonia or other conditions (8). This patient had no anticoagulated or antiplatelet drugs history previously. As she had no long-term history of low-grade fever, weight loss or cough, tuberculosis test for hemoptysis was not performed. However unusual symptoms, such as hemoptysis or atypical CT findings, may emerge in certain individuals, posing a significant challenge to the epidemic's management (9). In our case the patients had 
hemoptysis with other accompanying symptoms. In a recent study, hemoptysis was found to be 1.45-2.7 percent in the case of dengue fever, whereas in COVID-19 it was about 13 percent $(10,11)$. COVID-19 characteristics such as spike and nucleocapsid proteins activate the host's immune system, causing the virus to be eliminated. In the acute stage, these viral antigens can be identified by $\mathrm{B}$ cells or conveyed to $\mathrm{T}$ cells via MHC complexes, leading in antibody formation, enhanced cytokine secretion, and cytolytic activity (12). Increased blood levels of IL-2, IL-6, IL-10, and IFN- are signs of excessive cytokine production in severe instances of COVID-19 (13). High cytokine release and possible ACE2 expression downregulation in severe COVID-19 can not only promote a hypercoagulable condition separately, but also bilaterally boost each other's pro-thrombotic activities (13). Coagulation system activation is common in critically ill COVID19 patients (14). Hemoptysis may be an initial symptom of venous thromboembolism (VTE), and there is a case presenting with hemoptysis owing to pulmonary emboli associated with COVID-19 (3). In our patients, hypercoagulability values such as D-dimer and ferritin were within normal limits, and no VTE was observed in the radiological examination. Alveolar hemorrhage is a potential COVID-19 consequence in this case. Dengue is not known to cause lung alterations, and imaging results are likely due to increased vascular permeability. Dengue fever, hemoptysis, especially diffuse pulmonary infiltration needs to be evaluated in the differential diagnosis. Bilateral regions of ground-glass opacity or consolidation, as well as bilateral pleural effusions, are the most prevalent radiographic findings in dengue that also seen COVID-19 pneumonia (10). The key take away message from this case is that a lack of knowledge of hemoptysis as a potential COVID19 clinical manifestation has resulted in initial misdiagnosis and delayed diagnosis. When dealing with any patient arriving to the hospital in this pandemic situation with a history of hemoptysis, COVID-19 should be evaluated in the differential diagnosis list, even if the other characteristic clinical symptoms of COVID19, such as fever, dry cough, myalgia, and shortness of breath are missing. COVID-19 is a novel illness with many unknown features, unusual presentations such as severe hemoptysis should also arouse concern regarding the diagnosis of COVID-19, particularly in pandemic situations in dengue endemic settings.

\section{Conclusion}

In summary, COVID-19 should be considered as a differential diagnosis of acute undifferentiated febrile illness. In endemic regions, however, clinically suspected patients should be tested for both COVID-19 and Dengue fever, especially during the rainy season. This will aid in determining the burden of dengue and COVID-19 coinfection. Precise diagnosis is very crucial considering clinical presentation, exposure history and laboratory assays. During the COVID-19 pandemic, isolation of febrile ill patients' needs to be considered to, which leads to the prevention of disease transmission. COVID-19 can show with nonspecific symptoms; therefore, it is critical that physicians are aware of this potential. As a result, instances of COVID-19 with extrapulmonary and unusual manifestations, as well as co-infections such as dengue fever, should not be overlooked when patients present to hospital settings, where healthcare professionals should be cautious. We believe that a combination of these strategies will help Bangladesh deal with the COVID-19 and dengue virus outbreaks, lowering higher mortality rates and increasing the survival rate of COVID-19 cases, which may be intensified by dengue.

\section{Funding}

This research did not receive any specific grant from funding agencies in the public, commercial, or not-forprofit sectors. The authors are supported by other funding sources including the Bill and Melinda Gates Foundation.

\section{Declaration of competing interest}

The authors declare that they have no known competing financial interests or personal relationships that could have appeared to influence the work reported in this paper.

\section{Acknowledgements}

icddr,b is also grateful to the Governments of Bangladesh, Canada, Sweden and the UK for providing core/unrestricted support. 


\section{Consent}

The patient's written informed consent for publishing of this case report, as well as images, was acquired. The editor in chief of this journal has a copy of the written consent to review.

\section{Reference:}

1. Hossain I, Khan MH, Rahman MS, Mullick AR, Aktaruzzaman M. The epidemiological characteristics of an outbreak of 2019 novel coronavirus diseases (COVID-19) in Bangladesh: A descriptive study. Journal of Medical Science and Clinical Research. 2020;8(04).

2. Macera M, De Angelis G, Sagnelli C, Coppola N, COVID V. Clinical presentation of COVID-19: case series and review of the literature. Int J Environ Res Public Health. 2020;17(14):5062.

3. Casey K, Iteen A, Nicolini R, Auten J. COVID-19 pneumonia with hemoptysis: acute segmental pulmonary emboli associated with novel coronavirus infection. The American journal of emergency medicine. 2020;38(7):1544. e1-. e3.

4. Chowdhury PB, Hossain S, Biswas RK. A combination of COVID-19 and dengue fever in Bangladesh: Preparedness of Bangladesh. Journal of Global Health. 2020;10(2).

5. Dengue and severe dengue WHO | World Health Organization: WHO | World Health Organization; 2021 [cited 202110 july 2021]. Available from: https://www.who.int/news-room/fact-sheets/detail/dengue-andsevere-dengue.

6. Gagnon S, Quigley N, Dutau H, Delage A, Fortin M. Approach to hemoptysis in the modern era. Can Respir J. 2017;2017.

7. Singh V, Mishra SC, Singh P, Pradeepa A. Dengue Infection Induced Diffuse Alveolar Hemorrhage. Journal of Case Reports. 2020;10(2):68-71.

8. Ittrich H, Bockhorn M, Klose H, Simon M. The diagnosis and treatment of hemoptysis. Deutsches Arzteblatt International. 2017;114(21):371.

9. Shi F, Yu Q, Huang W, Tan C. 2019 novel coronavirus (COVID-19) pneumonia with hemoptysis as the initial symptom: CT and clinical features. Korean journal of radiology. 2020;21(5):537.

10. Marchiori E, Hochhegger B, Zanetti G. Pulmonary manifestations of dengue. Jornal Brasileiro de Pneumologia. 2020;46.

11. Mohan K, Nasimuddin S, Sathyaseelan RD, Meenakshisundaram PR, Gnanadesikan S, Malaiyan J. A study on clinical profile and laboratory parameters of dengue fever patients in Chennai, Tamil Nadu, India.

12. Shah VK, Firmal P, Alam A, Ganguly D, Chattopadhyay S. Overview of immune response during SARS-CoV-2 infection: lessons from the past. Front Immunol. 2020;11:1949.

13. Du F, Liu B, Zhang S. COVID-19: the role of excessive cytokine release and potential ACE2 downregulation in promoting hypercoagulable state associated with severe illness. J Thromb Thrombolysis. $2021 ; 51(2): 313-29$.

14. Mazzeffi MA, Chow JH, Tanaka K. COVID-19 associated hypercoagulability: manifestations, mechanisms, and management. Shock (Augusta, Ga). 2021;55(4):465. 


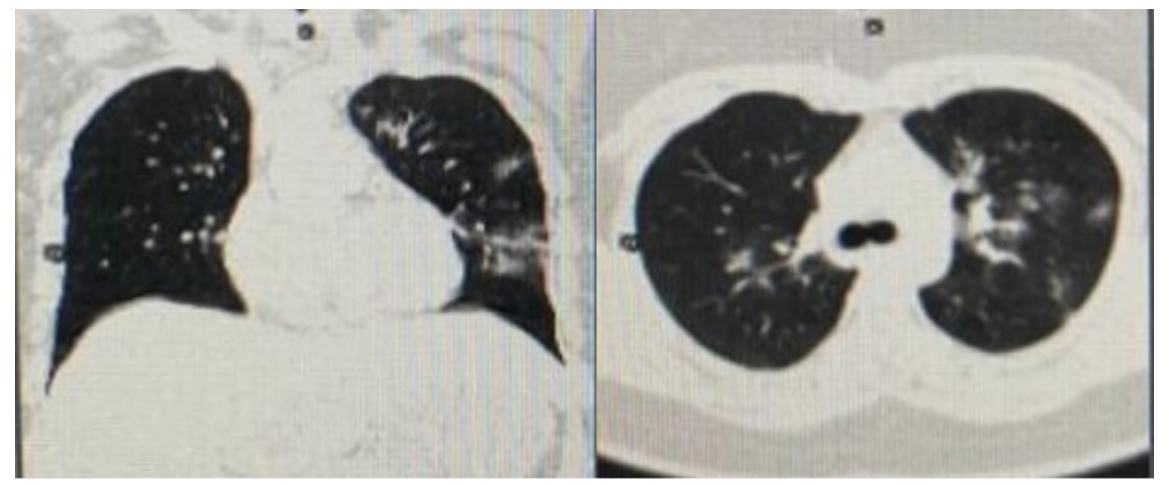

Figure 1. HR CT shows ground glass and consolidative opacities 